Ann. Biol. anim. Bioch. Biophys., I972, 12 (4), 58I-588.

\title{
ÉVOLUTION QUANTITATIVE ET QUALITATIVE DE LA CREATINE KINASE CHEZ LE LAPIN AU COURS DES PÉRIODES FGETALE ET NÉONATALE
}

\author{
F. BACOU \\ Station de Physiologie animale, \\ E. N. S. A., Place Viala, I. N. R. A., \\ 34060 Montpellier Cedex
}

RÉSUMÉ

Nous avons réalisé une étude sur l'évolution qualitative et quantitative de la créatine kinase chez le Lapin domestique, évolution qui n'était connue que par des données fragmentaires.

Ce travail a été effectué sur le fœtus total depuis l'âge de ro jours jusqu'à la naissance et sur le muscle Longissimus dorsi (long dorsal) depuis l'âge foetal de 24 jours jusqu'à 32 jours postpartum. L'évolution quantitative de cette enzyme chez le fœetus total se caractérise par une courbe analogue à celle observée dans le muscle long dorsal; l'étude qualitative a permis de mettre en évidence l'apparition des divers isoenzymes de la créatine kinase. Le fœtus de 16 jours ne contient que la forme BB, la forme MB apparaissant à 20 jours et la forme MM à 24 jours. Certains auteurs ont pu établir un parallèle entre les divers stades de formation des myofibrilles et l'apparition de ces divers isozymes, ceux-ci pouvant servir d'index de différenciation du tissu musculaire.

\section{INTRODUC'TION}

La créatine kinase (E. C. $2-7-3-2 ; C P K$ ) joue un rôle capital au cours de la contraction musculaire : elle permet le renouvellement en ATP, indispensable à la libération d'énergie, aux dépens de l'ADP et de la créatine phosphate.

Les travaux de DANCE et WATTS (I962); de DAwson et al. (I965) ont apporté la preuve de la nature dimérique de cette enzyme dont les protomères appartiennent à deux types : (B) et (M). La molécule de CPK existe donc sous la forme $\mathrm{BB}$, localisée essentiellement dans le cerveau, tandis que la forme $\mathrm{MM}$ représente le type musculaire. Enfin, ces protomères peuvent s'hybrider, formant le type MB que l'on rencontre surtout dans le cœur et parfois dans le muscle squelettique. 
Au cours de l'ontogenèse, le schéma isozymique du cerveau ne subit pas de modification; il n'en est pas de même des muscles squelettiques et du cour où l'on observe progressivement une diminution des isozymes BB et MB et une augmentation de l'isozyme MM. La mise en place du schéma adulte varie dans le temps selon les espèces animales : ainsi EPPENBERGER et al. (I964) montrent que l'évolution du schéma isozymique de muscle squelettique nécessite toute la vie embryonnaire et fœtale du Rat alors que les changements sont beaucoup plus rapides chez le Poulet. Chez 1'Homme, DAwson et FINE (I967) montrent la présence des trois isozymes chez le fotus humain de 6 semaines, tandis que Goto et al. (I969) chez le fœtus humain de deux mois ne découvrent que la forme $B B$.

EPPENBERGER et al. (I964) ont pu déduire quatre étapes du développement de la CPK dans le muscle squelettique du Poulet :

- la synthèse de CPK commence dès le myoblaste mais l'enzyme (uniquement sous forme BB) n'est pas utilisée car le substrat (la phosphocréatine) ne serait pas assez abondant.

- lors de l'apparition des protéines contractiles, l'enzyme, qui est sous forme MB, n'est pas encore utilisée à cattse de 1'absence (ou de l'insuffisance) de substrat.

- quand les myofibrilles apparaissent (dès le $r 3^{e}$ jour), on constate une augmentation rapide du taux d'actomyosine et de CPK dont le type MM est prépondérant. Le taux croissant de phosphocréatine indique que l'enzyme doit être active.

- à l'éclosion des poussins, il y a augmentation de la concentration en protéines spécifiques et en enzymes.

L'évolution de la créatine kinase chez le Lapin n'est connue que par des données fragmentaires (SchAPIRA et al., I968-I970) : nous avons donc entrepris une étude quantitative et qualitative de cette enzyme au cours de certains âges choisis pendant les périodes foetale et néonatale du Lapin domestique.

\section{MATÉRIEL, ET MÉTHODES}

Ces travaux ont été réalisés sur des lapins (communs et Néo-zélandais) élevés au laboratoire. Deux types d'études, quantitative et qualitative, ont été effectuées sur le fœtus total et sur le muscle long dorsal (Longissimus dorsi), choisi en raison de sa grande taille. Les âges retenus pour l'analyse quantitative et le nombre d'animaux par âge sont résumés dans le tableau $I$; les âges choisis pour l'analyse qualitative sont ceux illustrés dans la figure 3 .

\section{I. - Etude quantitative}

Les tissus musculaires ont été prélevés aussitôt après la mort des animaux et les fœetus recueillis dès le sacrifice de leur mère. Les échantillons qui n'ont pu être dosés immédiatement ont été mis au congélateur à $-30^{\circ} \mathrm{C}$ pendant $z$ à 3 jours au maximum.

L'enzyme a été extraite suivant une méthode dérivée de celle de Kumudavalli et al. (1970). Chaque échantillon a été homogénéisé à l'Ultra-Turrax dans trois fois son propre volume de tampon Tris $\mathrm{HCl}$ o, IM, pH 8,3 avec une $m$ Mole de EDTA. L'homogénat a été centrifugé à 35 ooo $g$ pendant 30 minutes et le surnageant utilisé pour le dosage. Toutes ces opérations se sont déroulées à $+4^{\circ} \mathrm{C}$. 
Pour le dosage, nous avons utilisé les "Biochemica tests combination CPK " (dosage dans l'UV à $34^{\circ} \mathrm{nm}$ ) de BOEHRINGER et SoEHNE dont la méthode est basée sur le couplage des réactions suivantes:

$$
\begin{aligned}
& \text { ATP + créatine } \stackrel{\mathrm{CPK}}{\longrightarrow} \text { ADP + créatine phosphate } \\
& \text { ADP + phosphoénolpyruvate } \stackrel{\text { PK }\left({ }^{1}\right)}{\longrightarrow} \text { ATP + pyruvate } \\
& \text { Pyruvate + NADH } \stackrel{\text { LDH }\left(^{2}\right)}{\longrightarrow} \text { lactate }+ \text { NAD }
\end{aligned}
$$

Nous avons suivi la réaction de phosphorylation de la créatine dans la cuvette maintenue à $37^{\circ} \mathrm{C}$ du spectrophotomètre enregistreur (Gilford 240). La diminuation de densité optique due à la transformation du NADH en NAD est proportionnelle à l'activité de la créatine kinase et matérialise la cinétique continue de la phosphorylation, permettant la détermination de l'activité enzymatique (d'après la tangente à l'origine). Celle-ci est exprimée en unités internationales ( $\mu$ moles de créatine phosphorylée par minute et par gramme de tissu frais).

\section{2. - Etude qualitative}

L'électrophorèse à $\mathrm{pH}$ alcalin permet de séparer les trois formes isozymiques de la créatine kinase : le type BB est attiré par l'anode beaucoup plus rapidement que le type MM, l'hybride MB ayant une vitesse intermśdiaire (Dawson et al., I968, SchapIRA, 1970; Kumudavalli et Watts, I968; HaNoune et Schapira, I966).

La méthode d'extraction de l'enzyme est identique à la précédente; le surnageant obtenu après centrifugation est ensuite concentré sous pression dans une cellule à ultrafiltration Amicon (ChARM et LAI., I97I).

Les électrophorèses ont été faites à $+4^{\circ} \mathrm{C}$ sur bandes d'acétate de cellulose (cellogel RS triangulaire) en utilisant un tampon Tris véronal, force ionique 0,05 et $\mathrm{pH} 8,8$; nous avons choisi un temps de migration de 4 heures sous une d.d.p. de 300 volts et une tension de Io $\mathrm{mA}$.

La technique de révélation utilise les réactions suivantes (DAWSon et al., 1965).

$$
\begin{aligned}
& \text { Créatine phosphate }+\mathrm{ADP} \stackrel{\mathrm{CPK}}{\longrightarrow} \text { créatine }+\mathrm{ATP} \\
& \text { ATP }+ \text { glucose } \stackrel{\text { hexokinase }}{\longrightarrow} \text { glucose } 6 \text { - phosphate }+ \text { ADP } \\
& \text { Glucose } 6 \text { - phosphate }+\mathrm{NADP} \stackrel{\mathrm{G}_{6} \mathrm{PDH}\left(\mathbf{(}^{3}\right)}{\longrightarrow} 6 \text {-phosphogluconate + NADPH. }
\end{aligned}
$$

Le phénazine méthosulfate sert d'intermédiaire dans le transport de l'hydrogène qui est transféré depuis le NADPH sur le nitrobleu de tétrazolium. Celui-ci apparait alors sur la bande de cellogel sous la forme d'un précipité bleu violacé.

\section{RÉSULTATS}

\section{I. - Étude quantitative}

Le travail effectué au cours de la période fœtale du Lapin sur le fœtus total montre la présence de créatine kinase dès le ro jour (tabl. I $a$ ). L'évolution du taux de cette enzyme peut se décomposer en deux parties : une élévation lente et progressive jusqu'à 20 jours suivie d'une brutale augmentation jusqu'à 28 jours (fig. I et tabl. I $a)$.

Si nous considérons l'évolution périnatale du muscle long dorsal étudié depuis 1'âge fœtal de 24 jours jusqu'à 32 jours post-partum (fig. 2 et tabl. I $b$ ), nous consta-

(1) PK : Pyruvate kinase.

(2) LDH : Lactate déhydrogénase.

(3) $\mathrm{G}_{6} \mathrm{PDH}$ : Glucose 6-phosphate déhydrogénase. 
tons que le taux de CPK, évolue très peu avant la naissance, contrairement à ce que nous observons chez le fotus total.

C'est après la naissance qu'une augmentation nette du taux d'enzyme se manifeste et ce jusqu'à I2 jours pour se stabiliser par la suite.

TABLEAU I

Activité de la créatine kinase selon les âges choisis

a) Fotus total

\begin{tabular}{c|c|c|c|c|c|c}
\hline Age (jours) & $10^{*}$ & $16^{*}$ & $20^{*}$ & $24^{*}$ & $28^{*}$ & 31 naiss. \\
\hline $\begin{array}{c}\text { Nbre d'animaux } \\
\text { Activité CPK } \\
\text { Écart-type }\end{array}$ & 25 & 4 & 4 & 5 & 5 & 5 \\
\hline & 1,65 & $\begin{array}{r}5,13 \\
1,34\end{array}$ & $\begin{array}{r}4,24 \\
2,31\end{array}$ & $\begin{array}{r}11,52 \\
1,63\end{array}$ & $\begin{array}{r}20,57 \\
0,81\end{array}$ & $\begin{array}{r}21,74 \\
5,60\end{array}$ \\
\hline
\end{tabular}

b) Muscle Long dorsal

\begin{tabular}{|c|c|c|c|c|c|c|c|c|c|}
\hline Age (jours) & $24^{*}$ & $28^{*}$ & 31 naiss. & 4 & 8 & 12 & 16 & 24 & 32 \\
\hline Nbre d'animaux & 5 & 5 & 5 & 5 & 5 & 5 & 4 & 4 & 5 \\
\hline Activité CPK & 14,69 & 19,35 & 24 & 88,79 & 131,99 & 189,03 & 180,03 & 198,29 & 269,64 \\
\hline Écart-type & 2,05 & 2,30 & 6,19 & 6,81 & 27,11 & 25,37 & 42,64 & 34,20 & 28,13 \\
\hline
\end{tabular}

* Cesââes correspondent à la période prénatale.

\section{2. - Étude qualitative}

Nous avons mis en évidence les trois isozymes de la créatine kinase dans le cœur, le cerveau et le muscle squelettique (long dorsal) adultes : le cerveau ne contient uniquement que la forme $\mathrm{BB}$; le cœur possède les trois formes isozymiques, le type $\mathrm{BB}$ étant le moins marqué et MB étant intermédiaire; de même on peut déceler les trois isozymes dans le muscle squelettique (Ar, ARD et CABROL, I970) avec des proportions proches de celles du cœur (fig. $3 a$ ). Toutefois, ces variations isozymiques pourraient refléter une réalité qui ne serait qu'apparente, les différences catalytiques entre les formes MM, MB et BB entraînant des activités enzymatiques différentes. Mais compte tenu des valeurs des $\mathrm{Km}$ et des vitesses de réaction calculées par EPPENBERGER et al (I967), il nous a paru que l'intensité de coloration observée sur la bande d'électrophorèse était suffisante dans les conditions de l'expérience pour évaluer l'évolution réelle des différents isozymes de la créatine kinase. 
En complément de l'étude quantitative évoquée au paragraphe précédent, plusieurs séries d'électrophorèse ont été effectuées : une première série a été réalisée sur des fotus totaux afin de déceler le passage de la forme BB aux autres formes isozymiques (fig. $3 b$ ) : l'âge de I6 jours ne nous montre que la forme isozymique BB caractéristique de la créatine kinase fœetale et que de nombreux auteurs déjà cités ont décelé chez le Poussin, le Rat et l'Homme.

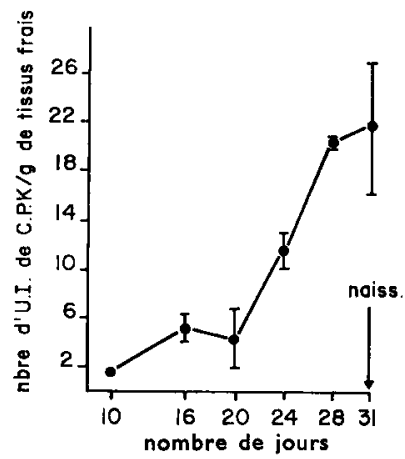

FIG. I. - Évolution quantitative de la créatine kinase chez le foetus total de Lapin domestique

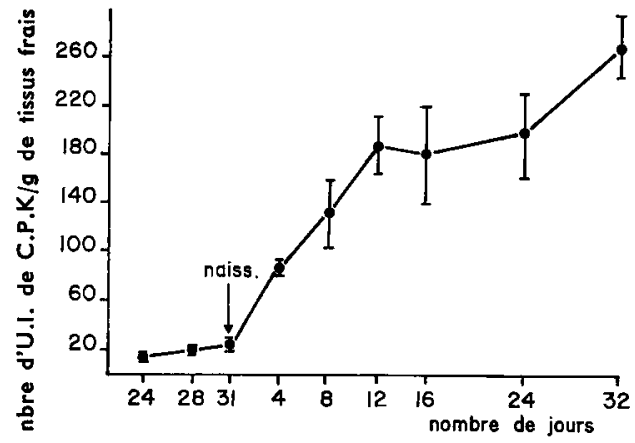

FIG. 2. - Évolution quantitative de la créatine kinase du muscle long dorsal au cours de la periode perinatale

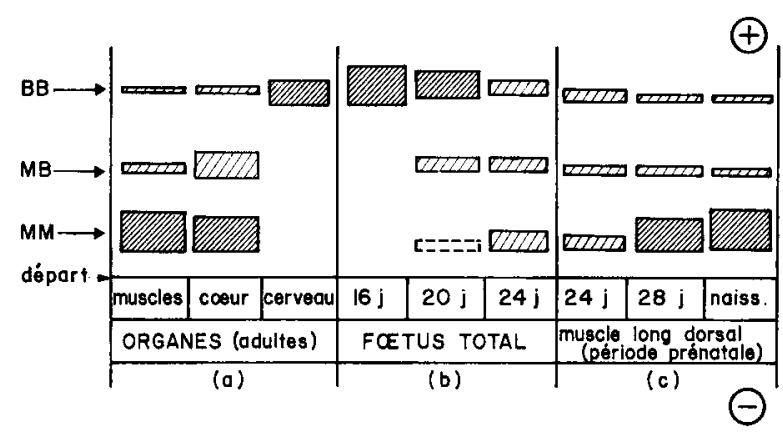

FıG. 3. - Évolution qualitative de la créatine kinase 
A l'âge de 20 jours, il y a apparition des bandes MB et même MM tandis que la forme BB régresse. Nous observons enfin une égalisation des bandes à 1'âge de 24 jours.

Une étude qualitative parallèle réalisée sur le long dorsal fœtal montre à 24 jours. un schéma isozymique semblable à celui obtenu au même âge chez le fœtus total (fig. $3 c$ ), c'est-à-dire la présence des trois isozymes avec une activité équivalente.

Nous avons ensuite, à 28 jours, un renforcement très net de la forme MM et une diminution de la forme BB. Le long dorsal à la naissance et aux stades suivants (non représentés sur la fig. $3 c$ ) montre enfin un schéma semblable à celui du muscle adulte.

\section{DISCUSSION}

Le muscle long dorsal montre une évolution quantitative relative à la CPK d'allure semblable à celle du fœtus total, mais décalée dans le temps. Si nous considérons l'ordre de précocité des régions musculaires du Lapin (VÉzINHET et al., I972), nous constatons que les muscles profonds du dos sont les plus tardifs : l'évolution pondérale se retrouve donc au niveau enzymatique.

Ce décalage dans le développement ne se retrouve pas au niveau qualitatif où le schéma isozymique du long dorsal est, chez le fœtus de 24 jours, semblable à celui du fœtus total au même âge. Cette similitude qualitative n'a rien d'étonnant puisque la musculature du fotus représente environ $40 \mathrm{p}$. Ioo de son poids total.

Les travaux réalisés sur les isoenzymes [Schaptra, I967 sur la LDH, la CPK et 1'aldolase; CAO et al. (Ig68-I97I) sur la CPK et la GOT (1)] concordent en un point : les schémas isozymiques évoluent pendant la vie fotale pour arriver, à un âge variable suivant les espèces, à un schéma semblable à celui de l'adulte. Selon AEBr et al. (1966), le schéma isozymique d'un organe dépend :

- de la nature des tissus,

- du degré de différenciation,

- de l'espèce.

Ceci est en accord avec les variations que nous avons pu observer sur les isozymes de la CPK du cerveau et du muscle adulte, d'une part, et des mêmes organes à un stade fœtal précoce, d'autre part.

Cependant la présence d'une enzyme ne prouve pas sa fonction. Si nous reprenons les quatre étapes définies par EPPENBERGER et al. (I964) sur le développement de la créatine kinase et décrites dans l'introduction, nous avons deux stades pendant lesquels l'enzyme existe mais ne peut être utilisée et les deux stades suivants où la présence du substrat en quantité adéquate permet son fonctionnement normal.

En nous reportant à nos propres résultats, nous constatons que la CPK évolue chez le Lapin suivant le même schéma que celui décrit par EPPENBERGE, chez le Poulet : jusqu'au $20^{\mathrm{e}}$ jour la concentration en enzymes croît lentement et la forme MM n'apparaît que très légèrement, la forme BB étant prépondérante. Par la suite nous constatons une augmentation brutale du taux d'enzymes, tandis que la forme MM. prend une part de plus en plus importante.

(1) GOT : Aspartate aminotransférase. 
Parmi les espèces (Lapin, Poulet, Rat...) étudiées par divers auteurs, la CPK semble évoluer de la même façon et parallèlement au degré de différenciation globale de l'animal. Toutefois, il est difficile d'expliquer le changement de type isozymique qui se manifeste dans le muscle et non dans le cerveau : selon EPPENBERGER $e t$ al. (1964) la succession des différents isozymes de la CPK dans le muscle serait associée au développement des structures hautement spécialisées contenant des protéines contractiles. Mais celles-ci ne doivent pas être les seules à intervenir puisque la musculature lisse a un schéma isozymique semblable à celui du cerveau (ALIARD et $\mathrm{C}_{\mathrm{ABROL}}$, I970). Les influences génétiques et nerveuses doivent aussi être considérées avant d'essayer de répondre à cette question.

Reģu pour publication en mai 1972.

\section{REMERCIEMENTS}

Je remercie J. LAFont, assistant au laboratoire de M. Moretri, Faculté des Sciences de Montpellier, pour les conseils techniques apportés à ce travail.

\section{SUMMARY}

QUANTITATIVE AND QUALITATIVE EVOLUTION

OF CREATINE, KINASE IN THE RABBIT DURING THE FOETAI, AND NEO-NATAL PERIODS

We made the following observations while studying the quantitative and qualitative evolution of creatine kinase during development in the rabbit :

- the enzyme is present on day Io of fœtal life (we do not know if it is used by the animal) It evolves slowly up to day 20 , then its activity suddenly increases up to day 28 . This period of foetal life (days 20-28) is thus marked by strong CPK synthesis ;

- while creatine kinase synthesis is slow in the long dorsal during rabbit foetal life, it rapidly increases after birth to level off at about day $\mathbf{2}$;

- creatine kinase (an isoenzyme having three forms) evolves qualitatively during growth, the I6-day total fotus showing only form $\mathrm{BB}$, and the 20 -day total foetus forms $\mathrm{BB}$ and $\mathrm{MB}$. At day 24, the fœtus has the three isoenzymic types: BB, MB, and MM. ;

- at pre-natal day 24, the long dorsal muscle has an isoenzymic plan resembling that of the total foetus at the same age. Later, forms MB and MM increase, the latter becoming predominant.

We have completed studies on Man, Rat, and Chicken by those which wereyet incomplete on the Rabbit. In studying this enzyme, primordial for muscle contraction, we have introduced a series of studies on the development of enzymes involved in muscle function and metabolism, thus adding to knowledge of muscle differentiation.

\section{RÉFÉRENCES BIBLIOGRAPHIQUES}

Aebi H., Richterich R., Wartburg Von J. P., r966. The isoenzyme pattern : some comparative biochemical and developmental aspects. Helgoländer wiss Meeresunters, 14, 343-351.

Allard D., Cabrol D., r97o. Étude électrophorétique des isozymes de la créatine phosphokinase dans les tissus de l'Homme et du Lapin. Path. Biol. Paris., 18, 847-850. 
Cao A., De Virgiliss S., Falorni A., r968. The ontogeny of creatine kinase isozymes. Biol. Neonat., 13, $375-380$.

Cao A., De Virgilis S., Lippi C., Coppa G., i971. Serum and muscle creatine kinase isoenzymes and serum aspartate aminotransferase isoenzymes in progressive muscular distrophy. Enzyme, 12, 49-62.

Charm S. E., LAI C. J., I97I. Comparison of ultrafiltration systems for concentration of biologicals. Biotech. Bioeng., 13, $185^{-202}$.

Dance N., Watrs D. C., I962. Comparison of creatine phosphotransferase from rabbit and brownhare muscle. Biochem. J., 84, i I p.

Dawson D. M., Eppenberger H. M., Eppenberger M. E., I 968 . Multiple molecular forms of creatine kinase. Ann. N. Y. Acad. Sci., 151, 6I6-626.

Dawson D. M., Eppenberger H. M., Kaplan N. O., 1965. Creatine kinase : evidence for a dimeric structure. Biochem. Biophys. Res. Commun., 21, 346-353.

Dawson D. M., FINE I. H., I967. Creatine kinase in human tissues. Arch. Neurol., 16, I75-180.

Eppenberger H. M., Eppenberger M., Richterich R., Aebi H., 1964. The ontogeny of creatine kinase isozymes. Devl. Biol., 10, 1-16.

Eppenberger H. M., Dawzon D. M., Kaplan N. O., I967. The comparative enzymology of creatine kinases. I. Isolation and characterization from chicken and rabbit tissues. J. biol. Chem., 242, $204-209$.

Goto I., Nagamine M., Katsukx S., I969. Creatine phosphokinase isozymes in muscles. Human fetus and patients. Arch. Neurol., 20,422-429.

Hanoune J., Schapira F., rg66. Action différente de l'insuline sur la créatine kinase du muscle et de cerveau de lapin. C. R. Soc. Biol., 160, 1807-1809.

Kumudavalli I., Moreland B. H., Watts D. C., I97o. Properties and reaction with iodacetamide of adenosine $5^{\prime}$-triphosphate-creatine phosphotransferase from human skeletal muscle. Further evidence about the role of the essential thiol group in relation to the mechanism of action. Biochem. J., 117, 513-523.

Kumudavalli I., Watts D. C., I968. Formation of an unusual hybrid in the development of human adenosine 5'-triphosphate-creatine phosphotransferase. Biochem. J., 108, 547-550.

Schapira F., r967. Pathological changes in the multiple molecular forms of LDH, Creatine kinase and aldolase. Bull. Soc. Chim. Biol., 49, 1647-I664.

Schapira F., 197o. Les isozymes de la créatine kinase et de l'aldolase. Path. Biol. Paris, 18, 295-3or.

Schapira F., Dreyfus J. C., Allard D., ig68. Les isozymes de la créatine kinase et de l'aldolase du muscle fotal et pathologique. Clinica chim. Acta, 20, 439-447.

Schapira F., Dreyfus J. C., Schapira G., I970. Ontogenic evolution and pathological modifications of molecular forms of some isoenzymes. Proc. Fedn Am. Soc. exp. Biol., 18, 305-320.

Vézinhet A., Rouvier R., Dulor J.-P., Cantier J., i972. Allométrie de croissance chez le Lapin. III. - Principales régions du système musculaire. Amn. Biol. anim. Biochim. Biophys. 12, 33-45. 